\title{
Mitochondria as a living system
}

\author{
Vlcek $\mathbf{M}^{*}$ \\ eFunctionality, Prague, Czech Republic
}

\section{Introduction}

The results of model work on economic systems are also used to understand the mitochondrial - bacterial system. The principle of the living system is extended by a more or less suspected structure of dark matter.

Dark matter as a rather astronomical term is interpreted as a fundamental part of the structure of living systems. Its function is connected with the materialization and control of the concept of existence. Recent research suggests that it may not be qualitatively different matter, but only matter that has fallen out of the existing system of normal matter. It is thus a world analogous but unobservable by normal means of normal matter.

\section{Living systems}

Living systems evaluate their existence on the basis of the satisfaction of their elements, and therefore on their (migratory) tendency to leave the system - the object. The degree of tendency is, in a certain simplification, the degree of existence [1].

The living object constantly evaluates the development of its own existence, and if it identifies an event known to it that announces the decline of existence, it activates the systemic means of reconstruction. They first evaluate the distribution of activities in the system and then activate the reconstruction part. As a result of its activities, there will be a change in the distribution of satisfaction and thus the required change in the existence of the object.

However, the living system thus described is only a part of it in normal matter. Dark matter then realizes its connection with other objects, in the realm of existence. Above all, due to dark matter, the living object is activated in the evaluation of its existence. The evaluation of the existence of a living object occurs only in times that are defined by dark matter, no universal time is available. Dark matter at this time acts as a pacemaker [2].

The feedback to dark matter is then the existence of one's own living system, which occurs when the object as a whole is activated. At the same time, the distribution of success and the reconstruction of relationships is evaluated so that through the reduction of stress in normal matter, the stress in satisfaction is also solved.

The object exists at the moment when its structure is activated in normal matter and also at this moment satisfaction is defined at all its levels. It is defined through this structure, and dark matter, through the abilities of its parts, is able to identify it.

\section{Mitochondrial system}

The building block of mitochondria is the water molecule. So everything revolves around this molecule, its satisfaction in the system is a basic criterion.

Under suitable conditions - salinity, temperature, and chemical composition - these water molecules combined and cooperated. Water as a living (!) Object has its motivational mechanism and this association was beneficial for it. Gradually there was a specialization all in normal, observable matter [3].

Within it, a group is formed that specializes in activities that are influenced not by observable events in normal matter, i.e. by the exchange and success of individual elements, but by their satisfaction. This is generally reflected in their tendency to leave the emerging system. These elements of the group thus gradually fall out of the normal world (matter) and approach dark matter - they cease to be observable. Their activity is a function of satisfaction - that is, the degree of existence and from the point of view of others in the group they become dead.

Because it is a group that specializes in generally needed activity, as they gradually die, they seem to surround the rest with their "torso". Alternatively, at least the rest permeate it. The group probably solves its function - monitoring migration and thus also its existence - in some cases by somehow limiting the movement of normal matter other elements. In analogous systems, this activity is called boundary formation.

The torso consists of the "remains" of dead elements and, above all, of their structure evaluating existence. This structure thus takes over its function from the original elements, in collaboration with dark matter. This decisive structure in the case of mitochondria is based on elements one level lower and these are little-guessed hydrogen-electronic structures.

The result is a living system whose building blocks - the elements - are water molecules that move in a "torsion of dead" molecules. This torso - a hydrogen-electronic structure - is gradually evolving and improving, but still only with the task of being able to better evaluate the "satisfaction" of the elements - water molecules.

\section{Conclusion}

Since the assumption of unity of not only material but also principles in different levels of organization of matter in mutual cooperation of normal and dark matter is made, it is possible to ask how transferable are possible experiences, procedures, events, intelligence between levels in general.

If so, it would be interesting to observe and get to know more distant systems and understand their life principles and use them in systems such as humans. This would probably be more useful than examining

${ }^{*}$ Correspondence to: Martin Vlcek, eFunctionality, Prague, Czech Republic E-Mail: martin.vlcek@efunctionality.eu

Received: March 21, 2020; Accepted: March 29, 2020; Published: April 05, 2020 
systems as close as possible, which are the easiest to understand but all the less beneficial.

However, it is probable that the interconnectedness of the existence of various living systems through dark matter is, among other things, the transfer of this intelligence between levels with a single goal - to maximize global existence.

\section{References}

1. Martin Vlček (2018) Dark matter in our ordinary life. Amazon in Wroclaw. ISBN 9781718882836.

2. Martin Vlček (2020) The existence. Clinical Reviews \& Cases 2: 1-2.

3. Martin Vlček (2020) Virus as a living object. American Journal of Virology and Diseases 2: 1-2.

Copyright: @2020 Vlcek M. This is an open-access article distributed under the terms of the Creative Commons Attribution License, which permits unrestricted use, distribution, and reproduction in any medium, provided the original author and source are credited. 